\title{
Electron density and carriers of the diffuse interstellar bands
}

\author{
P. Gnaciński ${ }^{1}$, J. K. Sikorski ${ }^{2}$, and G. A. Galazutdinov ${ }^{3}$
}

\author{
1 Institute of Theoretical Physics and Astrophysics, University of Gdańsk, ul. Wita Stwosza 57, 80-952 Gdańsk, Poland \\ e-mail: pg@iftia.univ.gda.pl \\ 2 Institute of Experimental Physics, University of Gdańsk, ul. Wita Stwosza 57, 80-952 Gdańsk, Poland \\ e-mail: fizjks@iftia.univ.gda.pl \\ 3 Korea Astronomy and Space Science Institute, 61-1 Whaam-dong, Yuseong-gu, Daejeon, 305-348, Republic of Korea \\ e-mail: gala@boao.re.kr
}

Received 10 January 2007 / Accepted 12 March 2007

\section{ABSTRACT}

\begin{abstract}
We have used the ionisation equilibrium equation to derive the electron density in interstellar clouds in the direction of 13 stars. A linear relation was found that allows determination of the electron density from the $\mathrm{Mg} \mathrm{I}$ and $\mathrm{Mg}$ II column densities in diffuse clouds. The comparison of a normalised equivalent width of 12 DIBs with the electron density shows that the DIBs equivalent width do not change with electron density varying in the range $n_{\mathrm{e}}=0.01 \div 2.5 \mathrm{~cm}^{-3}$. Therefore the DIBs carriers (1) can be observed only in one ionisation stage, or (2) the DIBs are arising in cloud regions (eg. cores or cloud coronas) for which we cannot determine the electron density.
\end{abstract}

Key words. ISM: clouds - ISM: molecules

\section{Introduction}

The diffuse interstellar bands (DIBs) are broad absorption features seen in the interstellar medium. There are almost 300 DIBs known in the optical and NIR spectrum (Galazutdinov et al. 2000). Despite over 80 years of investigations their nature is still unknown (for a review, see Herbig 1995). Many carriers have been proposed as carriers of DIBs, e.g. solid particles, simple molecules, negative atomic ions, carbon chains, and fullerens.

In 1985 van der Zwet \& Allamandola proposed polycyclic aromatic hydrocarbons (PAHs) as the source of DIBs. Since then it has been a popular hypothesis. There are however problems with obtaining gas-phase laboratory spectra of dehydrogenated and/or ionised PAHs to verify this hypothesis. Recently Cox \& Spaans (2006) have presented simulations of PAH charge-state distribution in various environments. In clouds with varying irradiation and density, the fractional abundances of PAH cations, neutrals, and anions change dramatically.

We used the ionisation equilibrium equation to obtain the electron densities in individual clouds. The electron density was compared to the equivalent width of DIBs and the $\mathrm{CH} / \mathrm{CH}+$ lines. The equivalent width of the $\mathrm{CH}+$ line drops with rising $n_{\mathrm{e}}$, but no changes were observed in the DIBs equivalent widths.

\section{Column densities and equivalent widths}

The aim of this paper was to check the dependences between the electron density and the carriers of DIBs. To determine the electron density we had to measure the column densities of two elements in two adjacent ionisation stages. Our target stars were stars that fulfill the following criteria:

- reddened stars of spectral type O or B;

- high-resolution Hubble Space Telescope (HST) spectra for at least $\mathrm{MgI}$ and $\mathrm{MgII}$ lines are available (both, GHRS and STIS spectra were used);
- hydrogen column densities are available;

- equivalent widths of the chosen DIBs are available.

The column densities of Mg I, Mg II, Si I, Si II, C II, and C II* were calculated from high-resolution HST spectra. The spectra from the ultraviolet spectral range were downloaded from the HST Data Archive. The GHRS spectra taken in the FP-SPLIT mode were processed with IRAF tasks poffsets and specalign to achieve the final spectrum. The column densities were derived using the profile-fitting technique, and the absorption lines were fitted by Voigt profiles. The transitions for which the natural dumping constant $(\Gamma)$ is not known (Mg II $1240 \AA$ doublet, Mg I $1828 \AA$ ) were fitted with a Gauss function. The cloud velocities $(v)$, Doppler broadening parameters $(b)$, and column densities $(N)$ for multiple absorption components were simultaneously fitted to the observed spectrum. Both lines of magnesium doublet (at $1200 \AA$ ) were also fitted simultaneously $-v, b$, and $N$ were common for both lines in the doublet. The wavelengths, oscillator strengths (f), and natural damping constants $(\Gamma)$ were adopted from Morton (2003).

A convolution with a point spread function (PSF) was also performed. The PSF for the GHRS spectrograph consists of two Gaussian components. The "core" Gaussian has a $F W H M=$ 1.05 diodes, while the "halo" component has $F W H M=5.0$ diodes (Spitzer \& Fitzpatrick 1993). The relative contribution of the "core" and "halo" components into the PSF is wavelengthdependent and was interpolated from the table in Cardelli et al. (1990). The Gaussian PSF for the STIS spectrograph depends on wavelength, slit, and the mode of observations. The tables with FWHM for the combination of mode and slit can be found in the "STIS Instrument Handbook" (Kim Quijano 2003). The $F W H M$ of the Gaussian PSF was wavelength-interpolated from these tables.

The derived column densities used to calculate the electron density are presented in Table 1 . The hydrogen (HI) column densities were adopted from Diplas \& Savage (1994). The 
Table 1. Column densities $\left(N\right.$ in $\left.\mathrm{cm}^{-2}\right)$ derived from the HST spectra.

\begin{tabular}{|c|c|c|c|c|c|c|c|c|c|c|c|}
\hline$\overline{\text { Star }}$ & $\overline{\overline{v^{a}}}$ & $\overline{N N(\mathrm{Mg} \mathrm{I})}$ & $\overline{b^{a}}$ & $\overline{N(\mathrm{Mg} \text { II })}$ & $\overline{b^{a}}$ & elem. & $\overline{\bar{N}}$ & $\overline{b^{a}}$ & elem. & $\overline{\bar{N}}$ & $\overline{b^{a}}$ \\
\hline HD 24534 & 18 & $7.2 \pm 0.4 e 13$ & 2.2 & $2.9 \pm 0.1 e 15$ & 3.3 & Si I & $9.3 \pm 0.6 e 11$ & 2.5 & Si II & $1.8 \pm 0.1 e 15$ & 3.2 \\
\hline HD 24912 & 14 & $4.9 \pm 0.6 e 12$ & 2.2 & $5.5 \pm 0.4 e 15$ & 5.3 & & & & & & \\
\hline HD 74455 & 25 & $8.6 \pm 0.3 e 12$ & 9.1 & $3.1 \pm 0.1 e 15$ & 5.3 & & & & & & \\
\hline HD 74455 & 5 & $1.7 \pm 0.1 e 12$ & 8.4 & $5.5 \pm 1.1 e 13$ & 14.2 & & & & & & \\
\hline HD 74455 & -160 & $4.2 \pm 0.2 e 11$ & 8.0 & $1.7 \pm 0.1 e 13$ & 9.9 & & & & & & \\
\hline HD 91316 & -8 & $4.2 \pm 0.6 e 12$ & 6.0 & $2.4 \pm 0.1 e 15$ & 6.1 & & & & & & \\
\hline HD 91316 & 17 & $1.8 \pm 0.4 e 12$ & 0.8 & $1.0 \pm 0.1 e 15$ & 5.6 & & & & & & \\
\hline HD 141637 & -7 & $7.7 \pm 0.1 e 12$ & 1.4 & $6.8 \pm 0.2 e 15$ & 1.5 & & & & & & \\
\hline HD 141637 & -12 & $2.8 \pm 0.1 e 12$ & 3.0 & $2.3 \pm 0.4 e 15$ & 5.1 & & & & & & \\
\hline HD 147165 & -9 & $1.4 \pm 0.2 e 13$ & 1.7 & $1.1 \pm 0.1 e 15$ & 4.2 & & & & & & \\
\hline HD 1 & -17 & $4.3 \pm 0.1 e 12$ & 2.7 & $2.1 \pm 0.1 e 15$ & 2.9 & & & & & & \\
\hline HD 1 & -29 & $3.9 \pm 1.0 e 11$ & 2.0 & $8.1 \pm$ & 2.8 & & & & & & \\
\hline HD 160578 & -27 & $1.1 \pm 0.3 e 11$ & 4.2 & $2.3 \pm 0.3 e 14$ & 5.3 & C II & $1.7 \pm 1.1 e 16$ & 3.6 & C II* & $8.0 \pm 1.3 e 12$ & 3.9 \\
\hline HD 202904 & -22 & $1.3 \pm 0.1 e 12$ & 4.2 & $3.6 \pm 1.9 e 14$ & 6.2 & C II & $8.4 \pm 7.4 e 15$ & 5.7 & C II* & $2.4 \pm 0.1 e 13$ & 2.9 \\
\hline HD 2 & -13 & $1.2 \pm 0.1 e 12$ & 2.5 & $1.2 \pm$ & 7.8 & C II & $1.8 \pm 0$ & 6.4 & C II* & $6.4 \pm 0$ & 5.1 \\
\hline HD 203374 & -18 & $1.2 \pm 0.1 e 14$ & 7.4 & $1.2 \pm 0.2 e 16$ & 9.6 & Si I & $1.8 \pm 0$. & 8.8 & Si II & $1.4 \pm 0.2 e 16$ & 8.9 \\
\hline HD 206267 & -13 & $1.7 \pm 0.1 e 14$ & 4.7 & $1.1 \pm 0.1 e 16$ & 7.3 & Si I & $2.3 \pm 0.2 e 12$ & 5.8 & Si II & $1.0 \pm 0.1 e 16$ & 6.4 \\
\hline HD 209339 & -15 & $1.2 \pm 0.1 e 14$ & 3.4 & $1.2 \pm 0.1 e 16$ & 7.1 & Si I & $1.2 \pm 0.3 e 12$ & 7.8 & Si II & $9.0 \pm 2.1 e 15$ & 6.9 \\
\hline HD 210839 & -31 & $2.9 \pm 0.3 e 13$ & 2.5 & $1.9 \pm 0.1 e 15$ & 7.0 & Si I & $4.9 \pm 1.5 e 11$ & 1.9 & Si II & $1.4 \pm 0.1 e 15$ & 5.8 \\
\hline HD 210839 & -13 & $8.5 \pm 0.1 e 13$ & 3.9 & $1.0 \pm 0.1 e 16$ & 6.5 & Si I & $1.3 \pm 0.1 e 12$ & 4.0 & Si II & $9.0 \pm 0.3 e 15$ & 7.6 \\
\hline
\end{tabular}

${ }^{a}$ Cloud velocities $(v)$ and Doppler broadening parameters $(b)$ are in $\mathrm{km} \mathrm{s}^{-1}$.

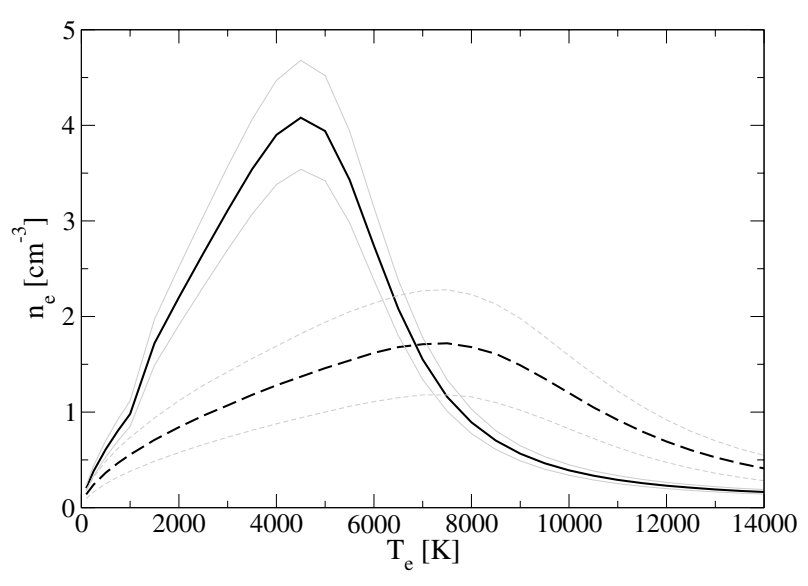

Fig. 1. The $n_{\mathrm{e}}\left(T_{\mathrm{e}}\right)$ curves from $\mathrm{MgI} / \mathrm{MgII}$ (solid line) and $\mathrm{SiI} / \mathrm{SiII}$ (dashed line) for the $v=-31 \mathrm{~km} \mathrm{~s}^{-1}$ cloud in the direction of HD 210839. The intersection point of these curves allows us to determine the electron density $\left(n_{\mathrm{e}}\right)$.

molecular hydrogen $\left(\mathrm{H}_{2}\right)$ column densities come from Rachford et al. (2002) and Savage et al. (1977). The equivalent widths of DIBs and $\mathrm{CH} / \mathrm{CH}+$ were kindly provided by Jacek Krełowski.

\section{Electron density}

The electron density $\left(n_{\mathrm{e}}\right.$ in $\left.\mathrm{cm}^{-3}\right)$ was calculated from the equations of ionisation equilibrium for two elements. The first element was $\mathrm{Mg}$, because it is easily observed in two ionisation stages. The $\mathrm{Mg}$ II column density was determined from the $1240 \AA$ doublet, and the Mg I column density was determined from the $2026 \AA, 2852 \AA$, or $1827 \AA$ line.

The step rise of dielectron recombination coefficient for $\mathrm{Mg}$ II with temperature causes the decrease in electron density, inferred from $\mathrm{MgI} / \mathrm{MgII}$, with temperature (Fig. 1). Such behaviour enables calculation of electron density, because the curve $n_{\mathrm{e}}\left(T_{\mathrm{e}}\right)$ from $\mathrm{Mg} \mathrm{I} / \mathrm{Mg}$ II intersects with a curve $n_{\mathrm{e}}\left(T_{\mathrm{e}}\right)$ from

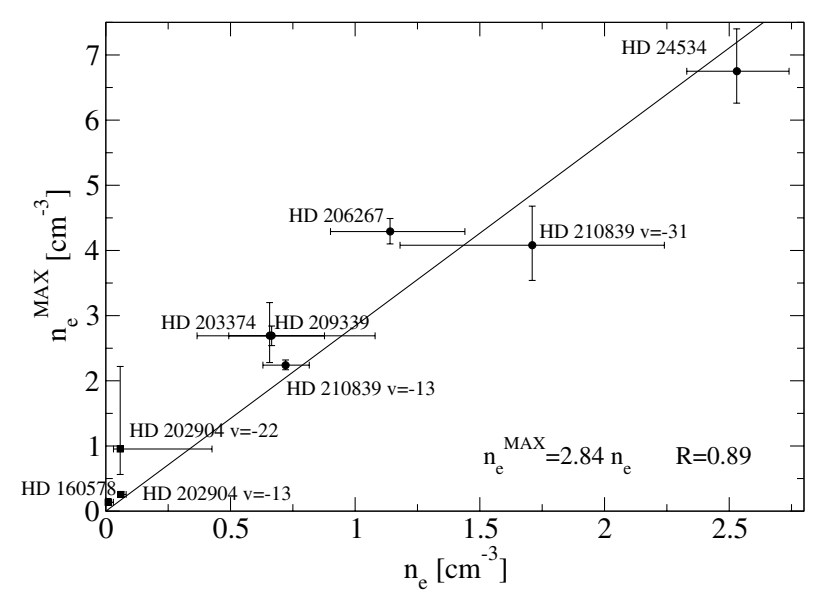

Fig. 2. The linear correlation between maximum $n_{\mathrm{e}}$ from the $N(\mathrm{MgII}) / N(\mathrm{MgI})$ curve and the exact $n_{\mathrm{e}}$ value.

another element. The equation of ionisation equilibrium for $\mathrm{Mg}$ is the following:

$\frac{n_{\mathrm{e}} N(\mathrm{Mg} \text { II })}{N(\mathrm{Mg} \mathrm{I})}=\frac{\Gamma\left(\mathrm{Mg}_{12}\right)+n_{\mathrm{e}} C\left(\mathrm{Mg}_{12}\right)}{\alpha_{\mathrm{rad}}\left(\mathrm{Mg}_{21}\right)+\alpha_{\mathrm{die}}\left(\mathrm{Mg}_{21}\right)}$,

where $N(\mathrm{MgII})$ and $N(\mathrm{MgI})\left[\mathrm{cm}^{-2}\right]$ are the column densities of ionised and neutral $\mathrm{Mg}, \alpha_{\mathrm{rad}}\left(\mathrm{Mg}_{21}\right)\left[\mathrm{cm}^{3} / \mathrm{s}\right]$ is the radiative recombination coefficient, $\alpha_{\text {die }}\left(\mathrm{Mg}_{21}\right)\left[\mathrm{cm}^{3} / \mathrm{s}\right]$ is the dielectronic recombination rate, $\Gamma\left(\mathrm{Mg}_{12}\right)[1 / \mathrm{s}]$ is the ionisation rate of $\mathrm{MgI}$ by UV photons, and $C\left(\mathrm{Mg}_{12}\right)\left[\mathrm{cm}^{3} / \mathrm{s}\right]$ is the collisional ionisation rate.

Because the coefficients $\alpha_{\text {rad }}, \alpha_{\text {die }}$, and $C$ depend on the electron temperature $\left(T_{\mathrm{e}}\right)$, we need an analogous equation for a second element to obtain $n_{\mathrm{e}}$ and $T_{\mathrm{e}}$ simultaneously. For the stars HD 24534, HD 203374, HD 206267, HD 209339, and HD 210839, the second element was Si. The column density of Si I was calculated from the $1845 \AA$ line, and the column density of Si II from the $1808 \AA$ one. From the intersection of the $n_{\mathrm{e}}(T)$ curves from $\mathrm{Mg}$ and $\mathrm{Si}$ (Fig. 1), we obtained the electron density. 
Table 2. Parameters used in the ionisation equilibrium equation.

\begin{tabular}{|c|c|c|c|c|}
\hline & $\begin{array}{c}\Gamma \\
{[1 / \mathrm{s}]} \\
\end{array}$ & $\begin{array}{c}C(T) \\
{\left[\mathrm{cm}^{3} / \mathrm{s}\right]} \\
\end{array}$ & $\begin{array}{l}\alpha_{\mathrm{rad}}(T) \\
{\left[\mathrm{cm}^{3} / \mathrm{s}\right]}\end{array}$ & $\begin{array}{l}\alpha_{\text {die }}(T) \\
{\left[\mathrm{cm}^{3} / \mathrm{s}\right]} \\
\end{array}$ \\
\hline $\mathrm{Mg} \mathrm{I} / \mathrm{II}$ & $8.1 \times 10^{-11}$ & $\begin{array}{l}8.9 \times 10^{-11} \sqrt{T}(1+0.1 \times T / 88700)^{-1} \\
\cdot \exp (-88700 / T)\end{array}$ & $\begin{array}{l}1.4 \times 10^{-13} \\
\cdot(T / 10000)^{-0.855}\end{array}$ & $\begin{array}{l}4.49 \times 10^{-4} T^{-3 / 2} \exp (-50100 / T) \\
\cdot(1+0.021 \cdot \exp (-28100 / T))\end{array}$ \\
\hline $\mathrm{Si} \mathrm{I/II}$ & $3.8 \times 10^{-9}$ & $\begin{array}{l}3.92 \times 10^{-10} \sqrt{T}(1+0.1 \cdot T / 94600)^{-1} \\
\cdot \exp (-94600 / T)\end{array}$ & $\begin{array}{l}5.9 \times 10^{-13} \\
\cdot(T / 10000)^{-0.601}\end{array}$ & $1.1 \times 10^{-3} T^{-3 / 2} \exp (-77000 / T)$ \\
\hline $\mathrm{Ca} \mathrm{I/II}$ & $3.8 \times 10^{-10}$ & $\begin{array}{l}2.09 \times 10^{-10} \sqrt{T}(1+0.1 \cdot T / 70900)^{-1} \\
\cdot \exp (-70900 / T)\end{array}$ & $\begin{array}{l}1.12 \times 10^{-13} \\
\cdot(T / 10000)^{-0.9}\end{array}$ & $\begin{array}{l}3.28 \times 10^{-4} T^{-3 / 2} \exp (-34600 / T) \\
\cdot(1+0.0907 \cdot \exp (-16400 / T))\end{array}$ \\
\hline $\mathrm{C}$ II/II* & - & $\begin{array}{l}8.63 \times 10^{-6}(2 \sqrt{T})^{-1} \Omega_{12}(T) \\
\cdot \exp \left(-\frac{1.31 \times 10^{-14} \mathrm{erg}}{k T}\right)\end{array}$ & $2.29 \times 10^{-6}$ & - \\
\hline
\end{tabular}

Note: the collision strength $\Omega_{12}(T)$ was fited by an 8-order polynomial to the data given by Hayes \& Nussbauer (1984).

Table 3. The electron densities derived for target stars.

\begin{tabular}{lrrrrrrrrr}
\hline \hline Star & $\begin{array}{r}v \\
{\left[\mathrm{~km} \mathrm{~s}^{-1}\right]}\end{array}$ & $\begin{array}{r}n_{\mathrm{e}} \\
{\left[\mathrm{cm}^{-3}\right]}\end{array}$ & $\begin{array}{r}n_{\mathrm{e}}^{\mathrm{MAX}} \\
{\left[\mathrm{cm}^{-3}\right]}\end{array}$ & $\begin{array}{r}\mathrm{HI} \\
{[\log N(\mathrm{HI})]}\end{array}$ & $\begin{array}{r}\mathrm{ref}^{a} \\
{\left[\log N\left(\mathrm{H}_{2}\right)\right]}\end{array}$ & $\begin{array}{r}\mathrm{CH}^{a} \\
{[\mathrm{~m} \AA]}\end{array}$ & $\begin{array}{r}\mathrm{CH}^{b} \\
{[\mathrm{~m} \AA}\end{array}$ \\
\hline HD 24534 & 18 & $2.5_{-0.2}^{+0.2}$ & $6.7_{-0.6}^{+0.6}$ & 20.73 & 3 & 20.92 & 1 & $3.2 \pm 0.4$ & $24.1 \pm 0.5$ \\
HD 160578 & -27 & $0.009_{-0.004}^{+0.02}$ & $0.14_{-0.05}^{+0.06}$ & 20.19 & 3 & & & & \\
HD 202904 & -22 & $0.06_{-0.03}^{+0.38}$ & $0.9_{-0.4}^{+1.3}$ & 20.68 & 4 & 19.15 & 4 & & \\
HD 202904 & -13 & $0.06_{-0.01}^{+0.02}$ & $0.26_{-0.04}^{+0.04}$ & & & & & & \\
HD 203374 & -18 & $0.66_{-0.16}^{+0.22}$ & $2.7_{-0.4}^{+0.5}$ & & & & & & \\
HD 206267 & -13 & $1.1_{-0.2}^{+0.3}$ & $4.3_{-0.2}^{+0.2}$ & 21.30 & 5 & 20.86 & 1 & $11.3 \pm 0.8$ & $21.7 \pm 0.9$ \\
HD 209339 & -15 & $0.7_{-0.3}^{+0.4}$ & $2.7_{-0.2}^{+0.2}$ & & & & & & \\
HD 210839 & -31 & $1.7_{-0.5}^{+0.5}$ & $4.1_{-0.5}^{+0.6}$ & 21.15 & 3 & 20.84 & 1 & $11.3 \pm 0.8$ & $22.3 \pm 0.4$ \\
HD 210839 & -13 & $0.72_{-0.09}^{+0.1}$ & $2.24_{-0.07}^{+0.08}$ & & & & & & \\
\hline
\end{tabular}

\begin{tabular}{|c|c|c|c|c|c|c|c|c|c|}
\hline HD 24912 & 14 & $0.09_{-0.02}^{+0.02}$ & $0.24_{-0.04}^{+0.05}$ & 21.05 & 3 & 20.53 & 2 & $21.13 \pm 0.19$ & $10.1 \pm 0.3$ \\
\hline HD 74455 & 25 & $0.27_{-0.02}^{+0.02}$ & $0.76_{-0.04}^{+0.05}$ & 20.73 & 3 & 19.74 & 6 & $1.0 \pm 0.3$ & $1.9 \pm 0.5$ \\
\hline HD 74455 & 5 & $3.0_{-0.6}^{+0.9}$ & $8.6_{-1.7}^{+2.6}$ & & & & & & \\
\hline HD 74455 & -160 & $2.4_{-0.08}^{+0.09}$ & $6.7_{-0.2}^{+0.3}$ & & & & & & \\
\hline HD 91316 & -8 & $0.17_{-0.02}^{+0.02}$ & $0.48_{-0.07}^{+0.07}$ & 20.44 & 3 & 15.61 & 7 & & \\
\hline HD 91316 & 17 & $0.17_{-0.04}^{+0.04}$ & $0.5_{-0.1}^{+0.1}$ & & & & & & \\
\hline HD 141637 & -7 & $0.109_{-0.004}^{+0.005}$ & $0.31_{-0.01}^{+0.01}$ & 21.18 & 3 & 19.23 & 4 & & \\
\hline HD 141637 & -12 & $0.12_{-0.02}^{+0.02}$ & $0.33_{-0.05}^{+0.07}$ & & & & & & \\
\hline HD 147165 & -9 & $1.2_{-0.2}^{+0.3}$ & $3.5_{-0.6}^{+0.7}$ & 21.38 & 3 & 19.79 & 2 & 4.5 & 2.9 \\
\hline HD 149757 & -17 & $0.192_{-0.006}^{+0.005}$ & $0.54_{-0.02}^{+0.02}$ & 20.69 & 3 & 20.65 & 2 & 22.4 & 18.0 \\
\hline HD 149757 & -29 & $0.046_{-0.012}^{+0.013}$ & $0.13_{-0.04}^{+0.04}$ & & & & & & \\
\hline
\end{tabular}

${ }^{a}$ (1)-Rachford et al. (2002); (2)-Savage et al. (1977); (3)-Diplas \& Savage (1994); (4)-Jenkins et al. (1986); (5)-Lacour et al. (2005); (6)- N(H calculated from the formula: $N\left(\mathrm{H}_{2}\right)=2.9 e 19 \cdot \mathrm{W}(\mathrm{CH})$; (7) -Bohlin et al. (1978);

${ }^{b}$ Adopted from Krełowski et al. (1999) and Krełowski (priv. comm.), and include absorption for all doppler components.

The $\Gamma$ coefficients for $\mathrm{Mg}$ and $\mathrm{Si}$ were adopted from the WJ2 model (de Boer et al. 1973). The recombination coefficients $\left(\alpha_{\text {rad }}\right.$ and $\left.\alpha_{\text {die }}\right)$ and the collisional ionisation rate coefficient $(C)$ (see Table 2) were adopted from Shull \& van Steenberg (1982).

For the stars HD 202904 and HD 160578, the ionisation equilibrium was calculated from $\mathrm{MgI} / \mathrm{MgII}$ and $\mathrm{CII} / \mathrm{CII} *$. The column density of carbon was calculated from the C II $1335 \AA$ and C II* $1336 \AA$ lines using the profile fitting technique (see Gnaciński 2000, for details). The equilibrium between the collisional excitation and radiative de-excitation of ionised carbon is described by the equation (Wood \& Linsky 1997):

$\frac{N\left(\mathrm{C} \mathrm{II}^{*}\right)}{N(\mathrm{C} \mathrm{II})}=\frac{n_{\mathrm{e}} C\left(\mathrm{C}_{12}\right)}{\alpha_{\mathrm{rad}}\left(\mathrm{C}_{21}\right)}$.

The radiative de-excitation $\alpha_{\text {rad }}\left(\mathrm{C}_{21}\right)$ was adopted from Nussbaumer \& Storey (1981). The collision rate coefficient $C\left(\mathrm{C}_{12}\right)$ was adopted from Wood \& Linsky (1997) and Hayes \& Nussbauer (1984).

We also tried to use $\mathrm{Ca}$ as the second element for obtaining $n_{\mathrm{e}}$. Unfortuately, the $\mathrm{CaI} / \mathrm{CaII}$ ionisation equilibrium curve 
Table 4. Equivalent widths of the diffuse interstellar bands in m̊̊ (courtesy of Jacek Krełowski).

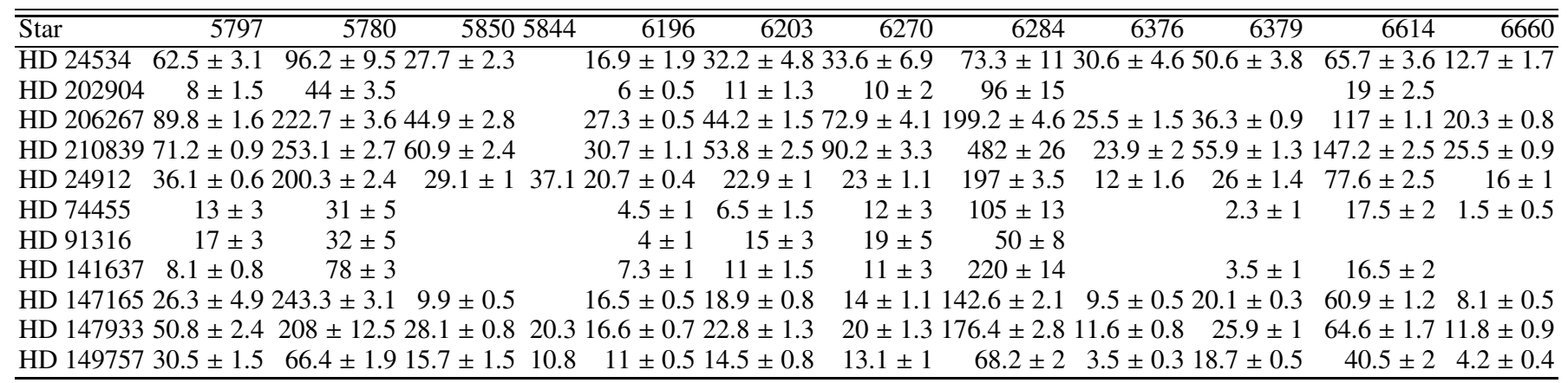
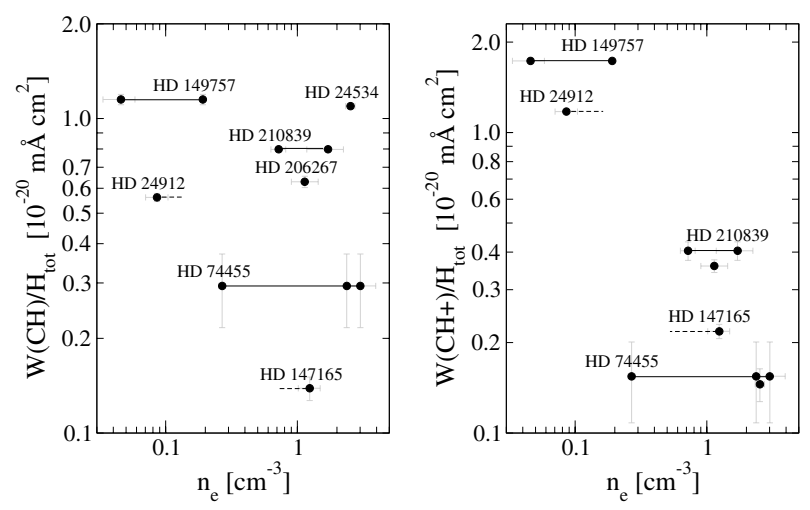

Fig. 3. The equivalent widths of the $\mathrm{CH}$ and $\mathrm{CH}+$ spectral lines normalised to the total hydrogen column density plotted versus electron density $\left(n_{\mathrm{e}}\right)$. The equivalent width of the $\mathrm{CH}$ and $\mathrm{CH}+$ lines includes all intervening doppler components. The solid lines connect points from different clouds in the direction of one star. The dashed lines indicate a second cloud with undeterminable electron density.

(Eq. (1)) for the stars HD 74455 and HD 149757 does not intersect the ionisation equilibrium curve for $\mathrm{Mg}$ I/II. The problem can be caused by the change of $n(\mathrm{CaII}) / n(\mathrm{CaI})$ between the edge and the centre of the cloud. In their numerical simulations Lepp et al. (1988) found that $n(\mathrm{CaII}) / n(\mathrm{CaI})$ decreases from 4800 at the edge to 160 at the centre of the $\zeta$ Persei cloud.

\section{Results and discussion}

For a lot of stars, $\mathrm{Mg}$ is the only element with observations of absorption lines of two stages of ionisation. From the column densities of neutral and ionised $\mathrm{Mg}$, we can only calculate the maximal possible electron density $n_{\mathrm{e}}^{\mathrm{MAX}}$. It is simply the maximum of the $n_{\mathrm{e}}\left(T_{\mathrm{e}}\right)$ curve. Fortunately this maximum $\left(n_{\mathrm{e}}^{\mathrm{MAX}}\right)$ is very well correlated with the electron density $n_{\mathrm{e}}$ (Fig. 2). The correlation coefficient $R=0.89$, and the linear correlation is $n_{\mathrm{e}}^{\mathrm{MAX}}=2.84 \cdot n_{\mathrm{e}}$. This relation was derived from $n_{\mathrm{e}}=0.01 \div 2.5 \mathrm{~cm}^{-3}$ and may not hold for denser or thinner environments. The linear relation $n_{\mathrm{e}}^{\mathrm{MAX}}=2.84 \cdot n_{\mathrm{e}}$ probably reflects that most of the clouds for which we can calculate $n_{\mathrm{e}}$ have the electron temperature $T_{\mathrm{e}} \sim 7500 \mathrm{~K}$. For stars HD 24912 , HD 74455, HD 91316, HD 141637, HD 147165, HD 147933, and HD 149757, the electron density was calculated using the $n_{\mathrm{e}}^{\mathrm{MAX}}$ value and this formula. All the derived electron densities are presented in Table 3.

Figure 3 presents the relation between the electron density and equivalent widths of the $\mathrm{CH}$ and $\mathrm{CH}+$ molecule normalised to the total hydrogen column density. The equivalent widths of $\mathrm{CH}$ and $\mathrm{CH}+$ include all doppler components. The $\mathrm{CH}$ abundance does not change between clouds with various electron

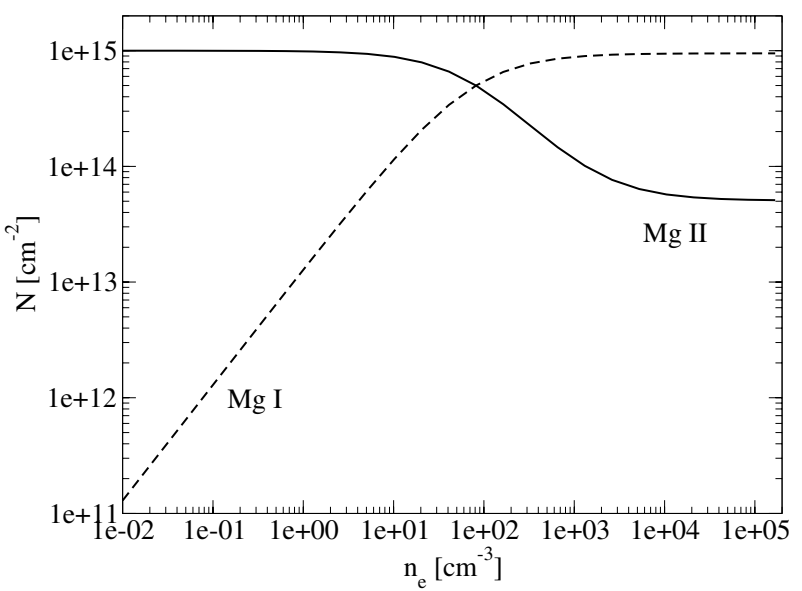

Fig. 4. Theoretical relation between the magnesium column density and the electron density $n_{\mathrm{e}}$. The solid line represents Mg II column density, while the dashed line represents $\mathrm{Mg}$ I column density.

density. In contrast to $\mathrm{CH}$, the $\mathrm{CH}+$ abundance is lower for clouds with high electron densities (more recombinations). Such behaviour is also illustrated in Fig. 4, where the theoretical relation between the column densities of $\mathrm{Mg}$ I and $\mathrm{Mg}$ II are presented versus the electron density.

We have checked that the changes in normalised $\mathrm{CH}+$ equivalent width with $n_{\mathrm{e}}$ are statistically significant. The points in Fig. 3 that are in the direction of the same star (connected with a straight line) were replaced by an average $n_{\mathrm{e}}$ value from two extreme points. The sample of stars was divided into two sets. One with $n_{\mathrm{e}}<0.4 \mathrm{~cm}^{-3}$ and the second one for directions with $n_{\mathrm{e}}>0.4 \mathrm{~cm}^{-3}$. We calculated the average $W(\mathrm{CH}) / \mathrm{H}_{\text {tot }}$ and $W(\mathrm{CH}+) / \mathrm{H}_{\mathrm{tot}}$ and their standard deviations for stars in both sets. The Student's t-variable was calculated in order to check the agreement between the averages for directions with $n_{\mathrm{e}}<$ $0.4 \mathrm{~cm}^{-3}$ and $n_{\mathrm{e}}>0.4 \mathrm{~cm}^{-3}$. The average of normalised $\mathrm{CH}$ for directions with low and high $n_{\mathrm{e}}$ agree with significance level 0.7. The average $W(\mathrm{CH}+) / \mathrm{H}_{\text {tot }}$ for directions with $n_{\mathrm{e}}<0.4 \mathrm{~cm}^{-3}$ and $n_{\mathrm{e}}>0.4 \mathrm{~cm}^{-3}$ differs substantially (significance level 0.009).

Figures 5-7 present equivalent widths of DIBs normalised to the total hydrogen column density $\left(N\left(\mathrm{H}_{\mathrm{tot}}\right)=N(\mathrm{HI})+2 N\left(\mathrm{H}_{2}\right)\right)$ plotted versus electron density $\left(n_{\mathrm{e}}\right.$ in $\left.\mathrm{cm}^{-3}\right)$. One could expect a drop in the DIBs equivalent width with $n_{\mathrm{e}}$ as seen in Fig. 3 for $\mathrm{CH}+$. Unfortunately, none of the DIBs bands show a relationship with varying electron density. There are two possible explanations for the lack of relationship between DIBs and $n_{\mathrm{e}}$. The first explanation that the carriers of the analysed DIBs may be observed only in one ionisation stage. In Fig. 4 we can see such behaviour for $\mathrm{Mg}$ II. In a wide range of observed electron 

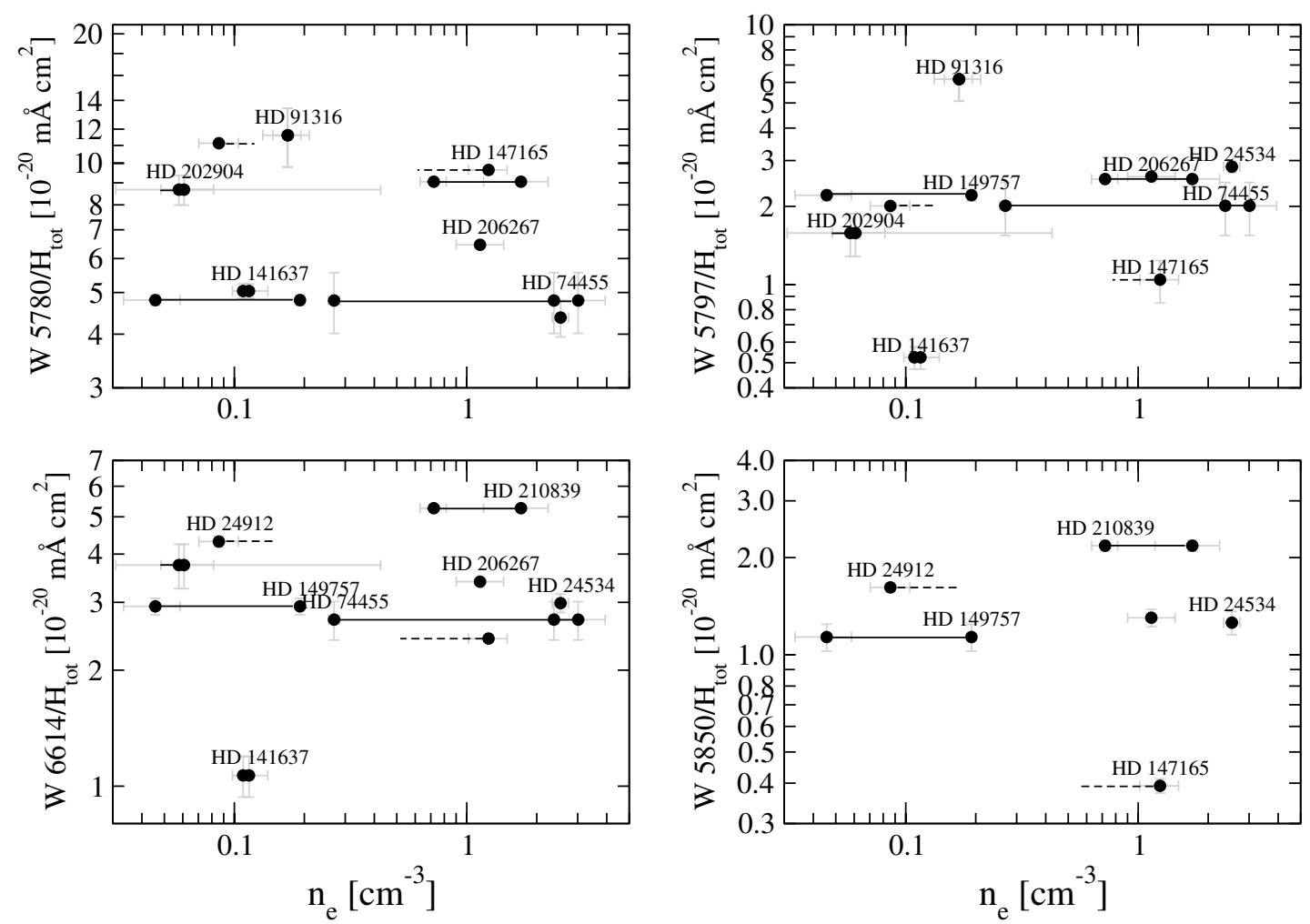

Fig. 5. The equivalent widths of DIBs normalised to the total hydrogen column density plotted versus electron density $\left(n_{\mathrm{e}}\right)$. The solid lines connect points from different clouds in the direction of one star. The dashed lines indicate a second cloud with undeterminable electron density.
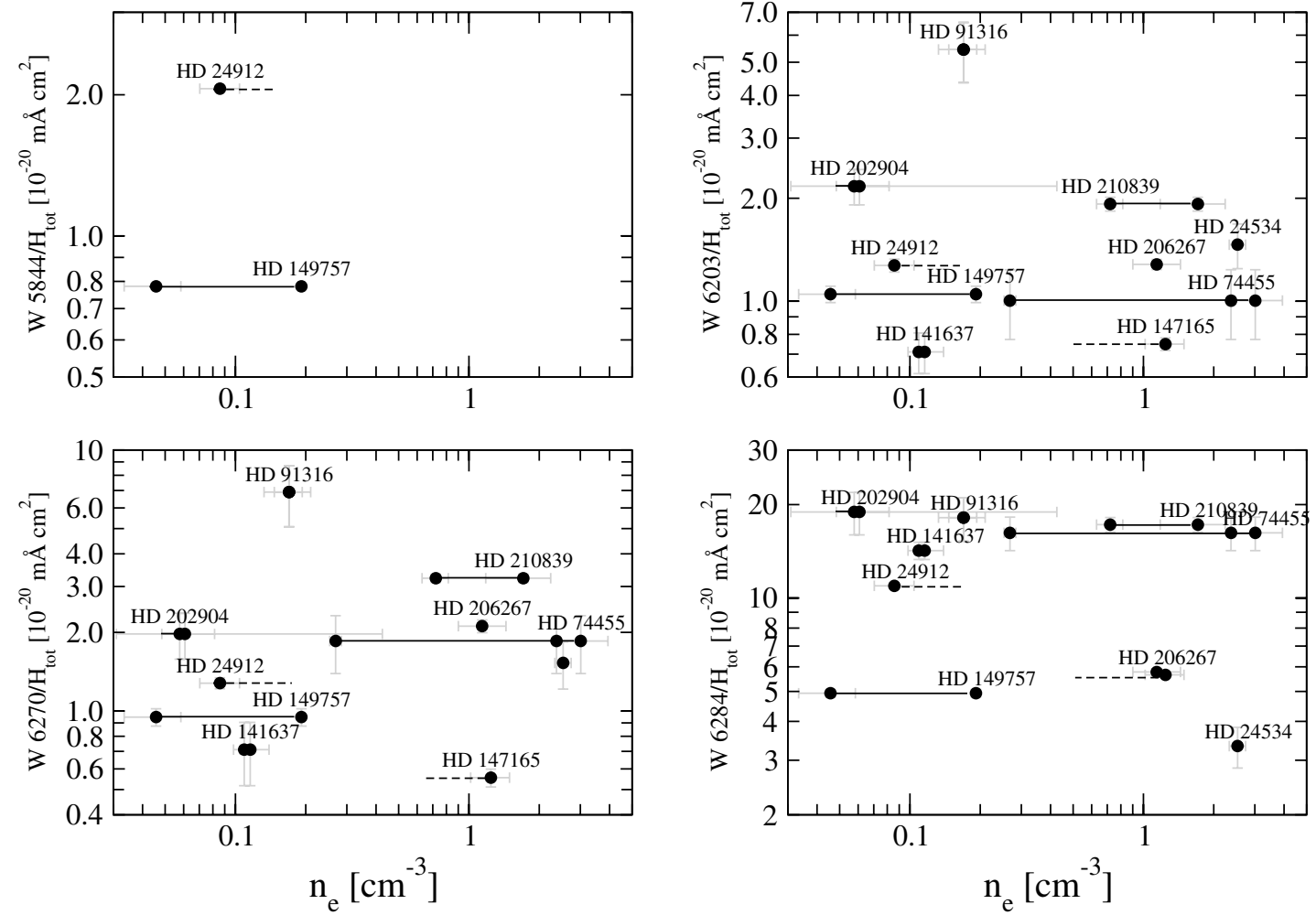

Fig. 6. The equivalent widths of DIBs normalised to the total hydrogen column density plotted versus electron density $\left(n_{\mathrm{e}}\right)$. The solid lines connect points from different clouds in the direction of one star. The dashed lines indicate a second cloud with undeterminable electron density.

densities $\left(n_{\mathrm{e}}=0.009-2.5 \mathrm{~cm}^{-3}\right)$, the column density of Mg II does not change by a considerable amount. Such behaviour is also seen for CH in Fig. 3.

The second explanation is that DIBs arise in parts of interstellar clouds where we observe only one stage of ionisation of
$\mathrm{Mg}$ and other elements. The DIBs can arise in dense cores of interstellar clouds, where ionised atoms are hardly observed. DIBs may also arise in outer (ionised) parts of the interstellar clouds, where neutral elements are absent. For both cases we cannot calculate the electron density. The hypothesis that DIBs carriers are 

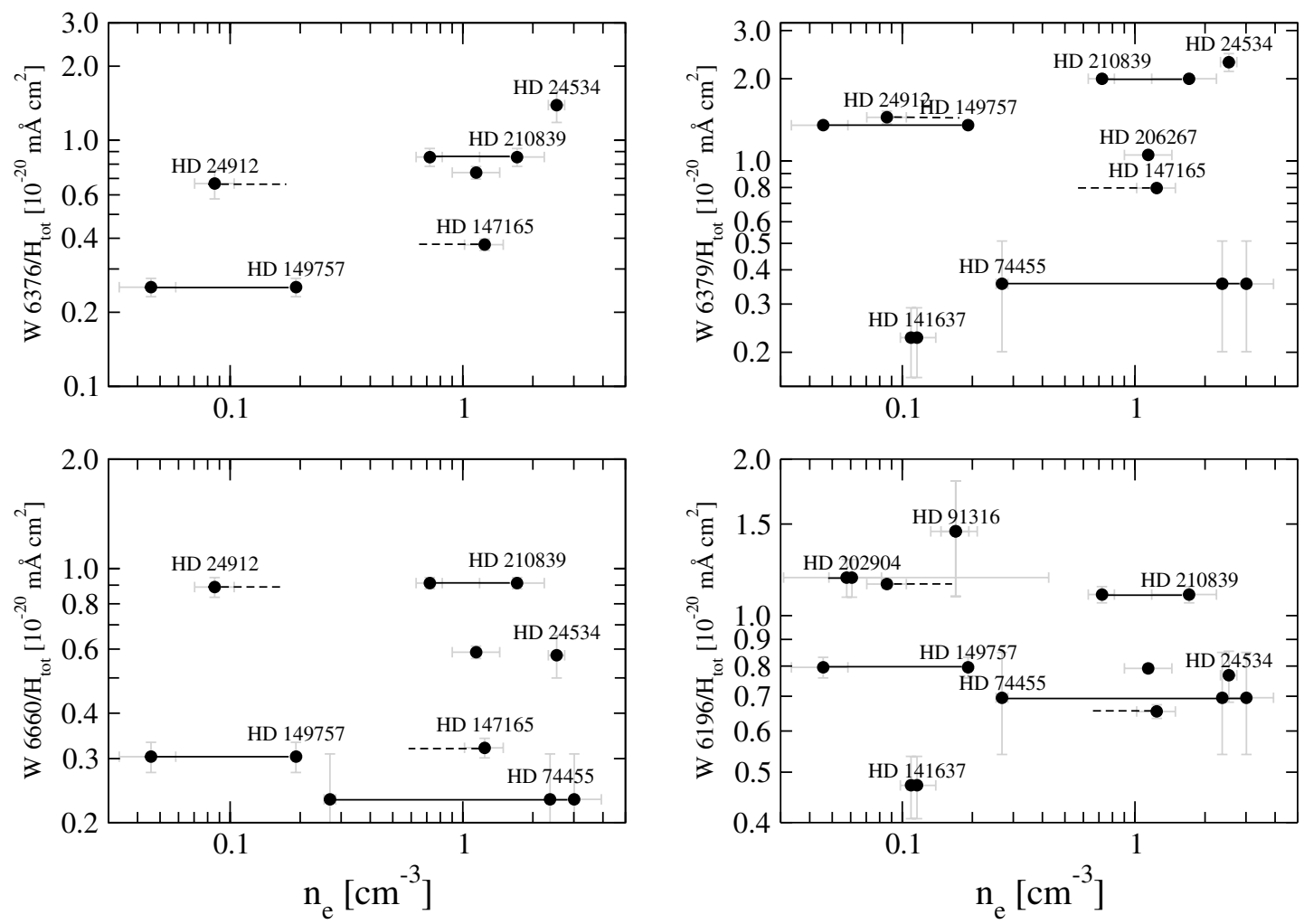

Fig. 7. The equivalent widths of DIBs normalised to the total hydrogen column density plotted versus electron density $\left(n_{\mathrm{e}}\right)$. The solid lines connect points from different clouds in the direction of one star. The dashed lines indicate a second cloud with undeterminable electron density.

formed in outer regions of interstellar clouds has already been formulated by Snow \& Cohen (1974). They observed that the $4430 \AA$ and $5780 \AA$ DIBs are shallower than expected in dense molecular clouds. This result was confirmed by observations of the Taurus dark clouds made by Adamson et al. (1991). High UV flux on the clouds surface may be responsible for ionising the DIBs carriers, while the cloud cores are shielded by extinction on dust grains.

\section{Conclusions}

The results can be recapitulated as follows.

1. The electron density in the interstellar clouds was determined for 13 lines of sight.

2. The linear correlation between the electron density and maximum possible value of electron density from $\mathrm{MgI} / \mathrm{MgII}$ was found to be $n_{\mathrm{e}}^{\mathrm{MAX}}=2.84 \cdot n_{\mathrm{e}}$.

3. The normalised equivalent width of the $\mathrm{CH}+$ line drops with rising electron density as expected from the ionisation equilibrium.

4. The normalised equivalent widths of the $5780 \AA, 5797 \AA$, $6614 \AA, 5850 \AA, 5844 \AA, 6203 \AA, 6270 \AA, 6284 \AA, 6376 \AA$, $6379 \AA, 6660 \AA$, and $6196 \AA$ DIBs do not change with electron density varying in the range $n_{\mathrm{e}}=0.01 \div 2.5 \mathrm{~cm}^{-3}$ (diffuse gas).

Acknowledgements. We are very grateful to Jacek Krełowski for the equivalent widths of the DIBs and the $\mathrm{CH} / \mathrm{CH}+$ lines. This publication is based on observations made with the NASA/ESA Hubble Space Telescope, obtained from the data archive at the Space Telescope Science Institute. STScI is operated by the Association of Universities for Research in Astronomy, Inc. under NASA contract NAS 5-26555.

\section{References}

Adamson, A. J., Whittet, D. C. B., \& Duley, W. W. 1991, MNRAS, 252, 234 de Boer, K. S., Koppenaal, K., \& Pottasch, S. R. 1973, A\&A, 28, 145 Bohlin, R. C., Savage, B. D., \&Drake, J. F. 1978, ApJ, 224, 132 Cami, J., Salama F., Jiménez-Vicente J., Galazutdinov G.A., \& Krełowski, J. 2004, ApJ, 611, L116

Cardelli, J. A., Ebbets, D. C., \& Savage, B. D. 1990, ApJ, 365, 789

Chlewicki, G., van der Zwet, G. P., nav Ijzendoorn, L. J., Greenberg, J. M., \& Alvarez, P. P. 1986, ApJ, 305, 455

Cox, N. L. J., \& Spaans, M. 2006, A\&A, 451, 973

Diplas, A., \& Savage, B. D. 1994, ApJS, 93, 211

Frisch, P. C., York, D. G., \& Fowler, J. R. 1987, ApJ, 320, 842

Galazutdinov, G. A., Moutou, C., Musaev, F. A., \& Krełowski, J. 2002, A\&A, 384,215

Galazutdinov, G. A., Musaev, F. A., Krełowski, J., \& Walker, G. A. H. 2000, PASP, 112,648

Gnaciński, P. 2000, Acta Astron., 50, 133

Hayes, M. A., \& Nussbauer, H. 1984, A\&A, 134, 193

Herbig, G. H. 1995, ARA\&A, 33, 19

Jenkins, E. B., Savage, B. D., \& Spitzer, L. 1986, ApJ, 301, 355

Kim Quijano, J., et al., 2003, STIS Instrument Handbook (Baltimore: STScI), available at http://www.stsci.edu/hst/stis/documents

Krełowski, J. 1989, Astron. Nachr., 310, 255

Krełowski, J., Ehrenfreund, P., Foing, B. H., et al. 1999, A\&A, 347, 235

Lepp, S., Dalgarno, A., van Dishoeck, E. F., \& Black, J. H. 1988, ApJ, 329, 418 Lacour, S., André, M. K., Sonnentrucker, P., et al. 2005, A\&A, 430, 967

Morton, D. C. 2003, ApJSS, 149, 205

Moutou, C., Krełowski, J., d'Hendecourt, L., \& Jamroszczak, J. 1999, A\&A, 351,680

Nussbaumer, H., \& Storey, P. J. 1981, A\&A, 96, 91

Omont, A. 1986, A\&A, 164, 159

Rachford, B. L., Snow, T. P., Tumlinson, et al. 2002, ApJ, 577, 221

Savage, B. D., Bohlin, R. C., Drake, J. F., \& Budich, W. 1977, ApJ, 216, 291

Shull, J. M., \& van Steenberg, M. 1982, ApJS, 48, 95

Snow, T. P. Jr., \& Cohen, J. G. 1974, ApJ, 194, 313

Sonnentrucker, P., Cami, J., Ehrenfreud, P., \& Foing, B. H. 1997, A\&A, 327, 1215

Spitzer, L. Jr., \& Fitzpatrick, E. L. 1993, ApJ, 409, 299

Weselak, T., Fulara, J., Schmidt, M. R., \& Krełowski, J. 2001, A\&A, 377, 677

Wood, B. E., \& Linsky, J. L. 1997, ApJ, 474, L39

van der Zwet, G. P., \& Allamandola, L. J. 1985, A\&A, 146, 76 